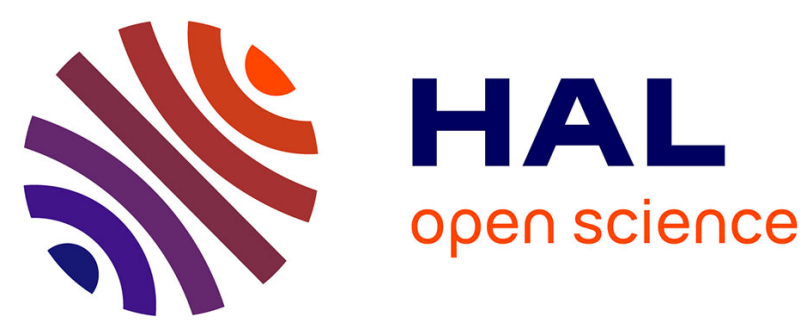

\title{
DIMENSIONAL METROLOGY OF FLEXIBLE PARTS: IDENTIFICATION OF GEOMETRICAL DEVIATIONS FROM OPTICAL MEASUREMENTS
}

Claire Lartigue, François Thiebaut, Pierre Bourdet, Nabil Anwer

\section{- To cite this version:}

Claire Lartigue, François Thiebaut, Pierre Bourdet, Nabil Anwer. DIMENSIONAL METROLOGY OF FLEXIBLE PARTS: IDENTIFICATION OF GEOMETRICAL DEVIATIONS FROM OPTICAL MEASUREMENTS. Advanced Mathematical and Computational Tools in Metrology VII, 2005, Caparica, Portugal. pp.196-203, 10.1142/9789812774187_0019 . hal-01226524

\section{HAL Id: hal-01226524 \\ https://hal.science/hal-01226524}

Submitted on 9 Nov 2015

HAL is a multi-disciplinary open access archive for the deposit and dissemination of scientific research documents, whether they are published or not. The documents may come from teaching and research institutions in France or abroad, or from public or private research centers.
L'archive ouverte pluridisciplinaire HAL, est destinée au dépôt et à la diffusion de documents scientifiques de niveau recherche, publiés ou non, émanant des établissements d'enseignement et de recherche français ou étrangers, des laboratoires publics ou privés. 


\title{
DIMENSIONAL METROLOGY OF FLEXIBLE PARTS: IDENTIFICATION OF GEOMETRICAL DEVIATIONS FROM OPTICAL MEASUREMENTS
}

\author{
CLAIRE LARTIGUE, FRANÇOIS THIEBAUT \\ IUT de Cachan, 9av. Division Leclerc, 94234 Cachan cedex - France \\ LURPA-ENS de Cachan-Université Paris sud-11 \\ PIERRE BOURDET \\ LURPA-ENS de Cachan-Université Paris sud-11 \\ 61 avenue du Pt Wilson, 94235 Cachan cedex - France \\ NABIL ANWER \\ IUT de Saint-Denis, \\ LURPA-ENS de Cachan-Université Paris sud-11
}

\begin{abstract}
This paper deals with an approach to identify geometrical deviations of flexible parts from optical measurements. Each step of the approach defines a specific issue to which we try to give an answer. The problem of measurement uncertainties is solved using an original filtering method, leading to only consider a few number of points. These points are registered on a mesh of the CAD model of the constrained geometry. From finite element simulation of the measuring set-up and of external forces, the shape resulting from deflection can be identified. Finally, geometrical deviations are obtained by subtracting geometrical deflections to measured geometrical deviations.
\end{abstract}

\section{Introduction}

Dimensional metrology of flexible parts is now a challenge, for the knowledge of part geometry is of a great importance in sheet metal assembly simulation. Sheet metal assembly consists in joining together one or more parts the characteristics of which are to be largely flexible and of complex forms. The process is often modelled as four sequential steps [1][2]:

- Parts are placed in fixtures.

- Parts are clamped.

- Parts are joined together.

- Assembly is released from the fixture. 
The simulation allowing understanding how dimensional variations propagate is based on a Finite Element Approach (FEA) which requires the finest definition of part geometry [1][2][3]. In fact, actual geometry deviations may affect boundary conditions as shown in figure 1 [2]: the displacement at the point Pt1 not only depends on the displacement DL1 but is also affected by the form deviation.

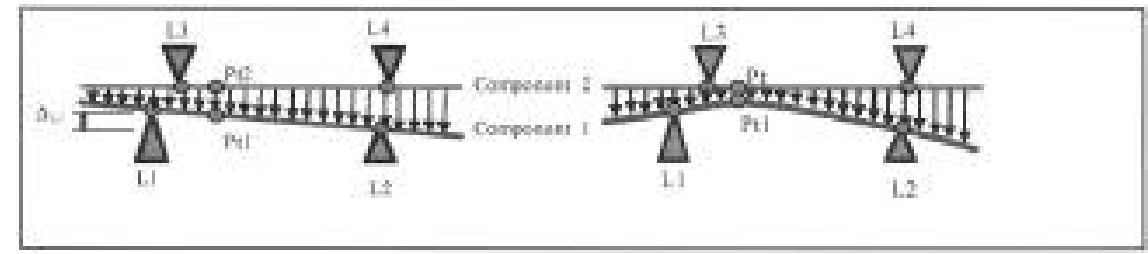

Figure 1: Effect of component shape in simulating the assembly process

More generally, the part geometry is modelled using a CAD system. Following, surfaces are meshed for the finite element calculation. In addition, real part surfaces are measured on inspection points using a classical measuring system: a CMM equipped with a contact probe. It is important to notice that to make simulation consistent, mesh nodes and inspection data must be coincident [2] [3]. As a result, the geometry of the surface is only acquired in a few measuring points which strongly depend on the initial meshing of the surfaces. This may affect the finite element simulation [2]. Moreover, as it is well-known that part set ups influence flexible plate parts' deflections under their own weight [4], parts are measured in the exact position of the assembly to integrate the weight effect. Therefore, classical methods for dimensional measurements of flexible parts are not yet satisfactory.

In this paper we propose an approach for dimensional measurements of flexible large-sized parts such as car doors. The successive steps leading to identifying geometrical deviations of flexible parts from optical measurements are proposed. Indeed, 3D optical digitizing systems are suitable for the measurement of large-sized flexible parts for they allow non-contact measurements and are able to deliver in a relatively short time large clouds of points that are representative of object surfaces.

The part is set-up on the CMM regardless of the assembly process. Due to its own weight and the supports, part deformations occur leading to displacements that can be of the same order than the geometrical deviations. 
Therefore, an identification method must be defined in order to extract geometrical deviations due to manufacturing defects only. However, it is here essential to detail some notions about the geometry of flexible parts as their shape may vary in function of their own weight.

\section{What is the geometry of a flexible part?}

The free shape of a part (component of an assembly) is the shape the part should have in the state of weightlessness. As this situation is rarely possible, the shape of a component is generally defined in the use state: when joined with other parts defining an over-constrained assembly subjected to external forces (figure 2). The use state defines the constrained geometry which is the support to the definition of the CAD model. It defines the reference geometry on which finite element calculations are conducted. When free from all the constraints, the shape of the component corresponds to the theoretical free shape: when the constraints are applied to the theoretical free shape, the geometry of the assembled component is identical to the CAD model. This theoretical free shape can be calculated from the FEA. By the way, the actual free shape is not identical to the theoretical free shape for it is not possible to elaborate exact geometry. It includes form deviations. At final, the component is measured defining the actual measured shape which includes deflections due to the part set-up and its own weight. These deflections can easily be simulated by the FEA.
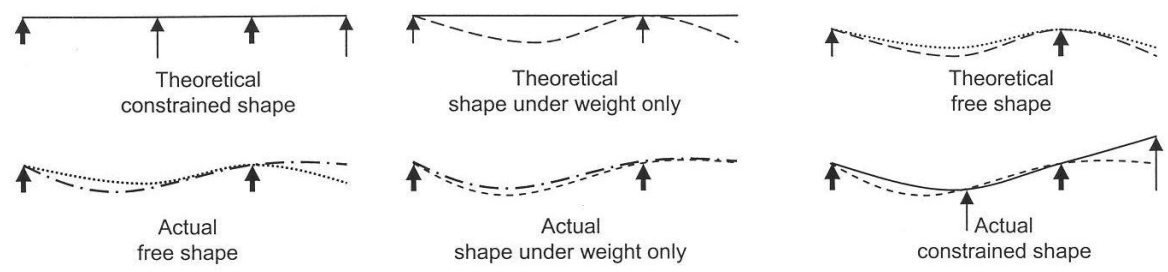

Figure 2: Various definitions of the geometry of compliant component for assembly

The standard ISO 10579 defines two ways of tolerancing flexible parts: tolerancing at the free-state and tolerancing under constraints. Indications must be the following: 
- Conditions of constraints: assembly, external forces (gravity).

- Admissible deviations at the free-state.

- Admissible deviations at the constrained state.

The free-state does not correspond to the state of weightlessness, considering that the position of the part as regards the direction of the gravity is clearly defined. However, tolerance values are greater in the free-state than in the constrained state which generally corresponds to the use state.

This section emphasizes difficulties in defining the shape geometry of flexible parts. Nevertheless, we suppose that a CAD model of the part surfaces exists.

\section{Optical measurements of flexible parts}

Optical measuring means seem more suitable to flexible part measurements than classical measuring means. The measuring system considered in this work is a CMM equipped with a motorized indexing head PH10 from Renishaw (http://www.renishaw.com), which supports a laser-plane sensor.

The part is set-up onto reference support points the position of which is clearly defined within the part frame. Note that the set-up must be non-over constrained allowing locating the frame part within the CMM framework. At this stage, the aptitude of the optical means with respect to dimensional measurements must be analyzed. As the scan planning influences measurement accuracy, the set of relative sensor/surface situations must be chosen so that local uncertainties are minimized [5]. Local indicators of quality can also be associated to points and compared to thresholds [5].

At this stage, the actual measured shape is defined as a large cloud of points which coordinates are defined in the CMM framework, $\mathrm{R}_{\mathrm{CMM}}$. The position of the locating points is also well-known in the CMM framework and defined by the rotation matrix $M$. The points are thus defined in the locating framework $R_{\text {loc }}$ by:

$$
O_{l o c} M_{i}=O_{l o c} O \mathbf{M} \quad . O M_{i}
$$

The restored cloud of points is discrete, inhomogeneous and dense. As data are further exploited, a pseudo-continuous representation of the points is carried out by a spatial $3 \mathrm{D}$ representation based on voxels (3D pixels). A voxel is a cube, the dimension of which is defined so that each cube at least contains a minimal 
number of measured points $\mathrm{M}_{\mathrm{i}}$ (figure 3). However, data must be filtered to remove effect of measure noise.

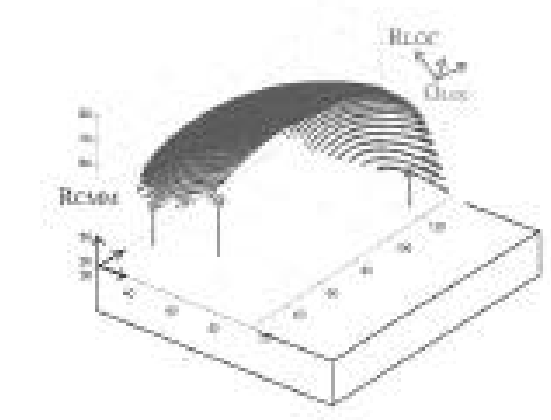

3ea Position of points and locating

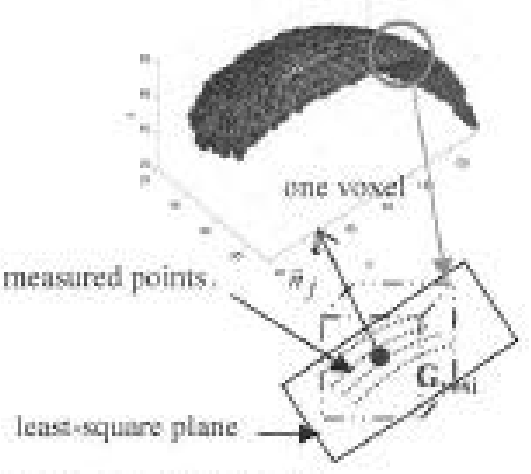

3-b voxel and is attribetes

Figure 3: Measured points and voxel representation

The barycentre of the measured points belonging to each voxel is calculated and the least-square plane is fitted to the points.

${\overrightarrow{O_{l o c} G}}_{v o x j}=\frac{\sum_{i=1}^{n_{v o x j}} w_{i} \cdot \vec{O}_{l o c} M_{i}}{\sum_{i=1}^{n_{\text {voxj }}} w_{i}}$

The normal to the plane defines the local normal $\dot{n}_{j}$ at the surface at the $\mathrm{G}_{\text {voxj }}$. A weight coefficient $\mathrm{w}_{\mathrm{i}}$ can be attributed to each point in function of its quality indicators. The orientation of each normal is outward material. Note that the definition of the voxel representation strongly depends on the surface mesh used for the FEA.

Following, the next step consists in the registration of the points on the CAD model in order to identify the actual free shape.

\section{Identification of the part geometry (free-state)}

The CAD model on which points must be registered is meshed according to the required mechanical calculations. Therefore, we have to register two clouds of points, one that results from the measurement and the other one which is linked 
to the meshing. For this purpose, we take advantage of the possibilities offered by the voxel representation and we use the Small Displacement Torsor (SDT) method [7].

Let $\mathrm{P}_{\mathrm{i}}$ be a theoretical point corresponding to a node of the mesh and $N_{i}$ be the local normal to the surface at the $\mathrm{P}_{\mathrm{i}}$ point. The problem is to find the corresponding point within the cloud of all measured points. Along the normal $N_{i}$, we move a voxel (cube the size of which is sufficiently small to represent a local neighbourhood of the mesh point) since it encounters a set of measured points. The barycentre of these points is calculated as well as the local normal to the voxel as discussed in the previous section: ( $\mathrm{Gi}, \dot{n}_{i}$ ) (figure 4 ).

Let $\xi i$ be the deviation between $\mathrm{G}_{\mathrm{i}}$ and $\mathrm{P}_{\mathrm{i}}$ projected onto the normal $N_{i}$ : $\xi_{i}=P_{i} G_{i} \cdot \dot{N}_{i}$ Let'( $\left.\dot{H}_{\mathcal{H}}\right)$ be the components of the SDT associated to the meshed surface, where $D_{A}$ is the displacement of a reference point and ${ }^{J}$ is the rotation vector. As the displacement of each point $\mathrm{P}_{\mathrm{i}}$ can be expressed using ( $D$ 丹 ), the optimized deviation can be expressed as follows:

$$
e_{i}=\xi_{i}-D_{P_{i}} \cdot N_{i}=\xi_{i}-\left(D_{A} \cdot N_{i}\right)-\left(\Omega \otimes A P_{i}\right) \cdot N_{i}
$$
Therefore, we have to find the coordinates of '( $\left.\dot{D}_{\mathcal{H}}\right)$ so that $\ldots \mathbf{L}_{i=1}^{-\imath}$ is
minimal [7], where $\mathrm{m}$ is the number of mesh points.

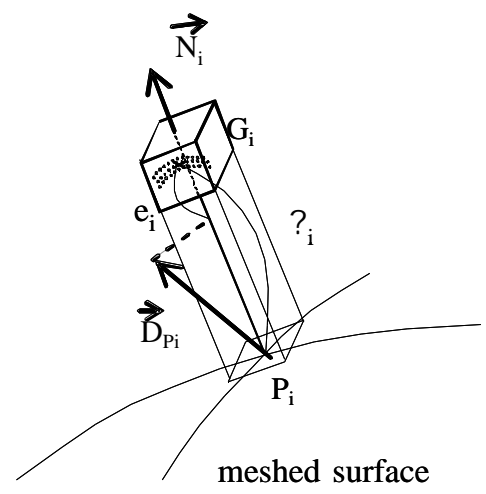

Figure 4: Point registration

As only barycentres are used for point registration, the effect of digitizing noise is clearly decreased. By the way, as all the measured points are conserved, form 
deviations can be evaluated for a large number of points.

The last step concerns the evaluation of geometrical deviations. The effect of part flexibility must be here taken into account. Linearity is assumed here, which leads to linearly separate the different sources of deviations as follows:

$$
\left[\mathrm{E}^{\mathrm{Form}}\right]=\left[\mathrm{E}^{\text {Measured }}\right]-\left[\mathrm{E}^{\mathrm{def}}\right]
$$

where $\left[\mathrm{E}^{\text {Measured }}\right]$ is calculated for each point of the mesh as the deviation between the CAD model and the measured surface according equation 3, and $\mathrm{E}^{\mathrm{def}}$ is the deviations due to part deflections. $E^{\text {def }}$ results from the simulation of the mechanical behaviour of the part (under its weight) located on the supports, and thus evaluate the displacements due to part deformation. As the mesh of the structure is obtained from the CAD model which is defined considering the constrained geometry, we have to first simulate the theoretical free shape using FEA. To simulate the mechanical behaviour, the relationship between the displacements and the forces $[\mathrm{F}]$ is assumed to be linear:

$$
[\mathrm{F}]=[\mathrm{K}][\mathrm{U}]
$$

where $[\mathrm{K}]$ is the rigidity matrix established through finite element modelling, assuming the material to be elastic and isotropic; [U] is the displacement vector and $[\mathrm{F}]$ is the force vector for all mesh knots [3]. In function of the chosen setup, either forces or displacements are imposed at the reference points. Inversing (5), after the two states are simulated, leads to $\left[\mathrm{E}^{\mathrm{def}}\right]$, the displacement vector associated to the deformation of the part when relaxed from its constrained and set-up for measurement. Note that, in most cases, industrial users prefer to measure in the use state in order to be free from mechanical simulations of the effect of the set-up.
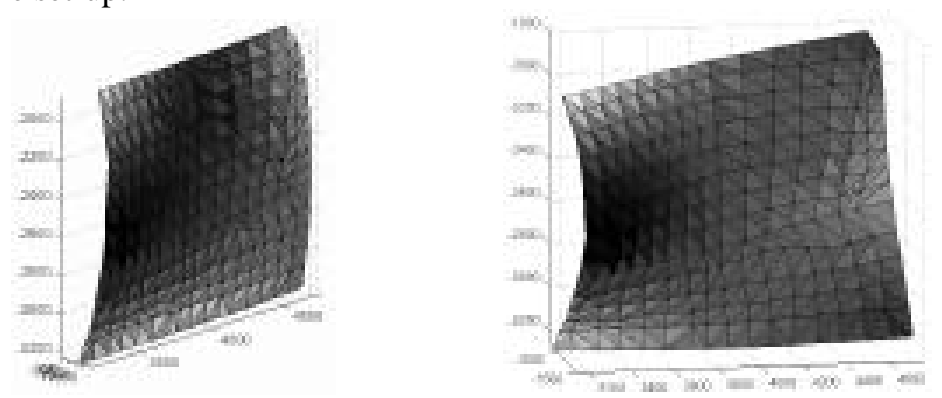

5 a boundary conditions on the theoretical shape 5 b boundary conditions on the actual shape

Figure 5: Influence of the form deviation on mechanical simulations 
At this stage, the form geometry of the part is known including form deviations. Simulation of the assembly process can be carried out taking into account the actual shape, as illustrated in figure 5, for which measured points are simulated. When boundary conditions are applied onto the actual shape, the mechanical behaviour may strongly vary [3]. The mesh is obviously preserved for both simulations.

\section{Conclusions}

Knowing the actual shape of a part which is a component of an assembly is essential to correctly simulate the assembly process. This paper has detailed the steps of an approach we propose to identify geometrical deviations of flexible parts from optical measurements. Each step of the approach leads to a specific problem to which we suggest some solutions. Measuring using an optical digitizing system gives large sets of noisy points. To decrease the effect of digitizing noise, we suggest an original filtering method by defining a voxel representation onto the points. The barycentre of the voxel is calculated as the barycentre of the points belonging to the voxel. This leads to only consider a few number of points which are registered on the node points of the mesh of the CAD model (the constrained geometry) using the SDT method. From finite element simulation of the measuring set-up and of external forces, the shape resulting from deflection can be identified. Finally, geometrical deviations are obtained by subtracting geometrical deflections to measured geometrical deviations. The development of this approach is actually in progress.

\section{References}

1. Camelio J., Jack Hu S., (2003), Modeling variation propagation of multistation assembly systems with compliant parts, J. of Mechanical design, 125 : 673-681

2. Dahlström S., Lindkvist L., Södeberg R., (2005), Practical implications in tolearance analysis of sheet metal assemblies. Experiences from an automotive application. CIRP seminar on Computer Aided Tolerancing, Charlotte (USA), CDRom paper

3. Cid G., Thiébaut F., Bourdet P., Falgarone, H., (2005), Geometrical study of assembly behaviour taking into account rigid components'deviations, actual geometric variations and deformations, CIRP seminar on Computer Aided Tolerancing, Charlotte (USA), CDRom paper 
4. Lee E.S., Burdekin M., (2001), A hole-plate artifact design for the volumetric error calibration of CMM, Int J Adv Manuf Technol, 17:508-515

5. Lartigue C., Contri A., Bourdet P., Digitised point quality in relation with point exploitation, Measurement 32, pp. 193-203, 2002

6. ISO Standard 10579 (1993), Tolérancement des pièces non rigides

7. Bourdet P., Clément A., (1976), Controlling a Complex Surface with a 3 axis measuring machine, Annals of the CIRP, 25(1):359-361 